\title{
History-Dependent Inference Control of Queries by Dynamic Policy Adaption*
}

\author{
Joachim Biskup \\ Technische Universität Dortmund, Dortmund, Germany \\ joachim.biskup@cs.tu-dortmund.de
}

\begin{abstract}
Policy-based inference control of queries submitted to a logicoriented information system requires us to consider the history of queries and answers to a particular user. In most previous approaches, the control system captures the history by maintaining a fictitious view the user is supposed to generate by exploiting rational reasoning. In this paper, we propose and explore an alternative option to represent the history, namely by suitably adapting the confidentiality policy after returning an answer to a query. Basically, such a policy adaption precomputes all relevant steps of formal proofs that the fictitious view logically implies some policy element. We focus on propositional information systems.
\end{abstract}

Keywords: a priori knowledge, closed query, confidentiality policy, Controlled Query Evaluation, inference control, information system, interaction history, policy adaption, propositional logic, refusal, view.

\section{Introduction}

Inference control is a crucial though costly mechanism to protect information rather than just the underlying data, as achieved by traditional access control or simple encryption [4. In general, dynamic inference control of queries submitted to an information system necessarily requires us to consider the history of queries and answers related to a particular user. In most of the previous work, including those on Controlled Query Evaluation (CQE) [5], the control employs the user's history in two ways: First, the control generates an (assumption about the) view that the user (supposingly) infers to represent his knowledge about the instance of the information system. This instance itself, however, remains hidden to the user, except that he has seen the previous answers and might have access to some a priori knowledge. Second, the control investigates whether that view combined with the correct answer to the next submitted query (or some closely related information) would be harmful w.r.t. a confidentiality policy specifically declared for the user.

In this context the intuitive meaning of harmful is the following: the user will be able to infer that some sentence contained in the policy actually holds in the

\footnotetext{
* This work has been performed within the framework of the Collaborative Research Center "Providing Information by Resource-Constrained Data Analysis", supported by the Deutsche Forschungsgemeinschaft under grant SFB 876/A5.
}

Y. Li (Ed.): Data and Applications Security and Privacy XXV, LNCS 6818, pp. 106-121, 2011.

(C) IFIP International Federation for Information Processing 2011 
instance. If this will be the case, the control reacts with a suitable distortion of the correct answer to avoid a security violation. In any case, after returning a reaction to the user, the control has to appropriately adjust the view generated for the user. Thus, over the time, the control enforces a suitable invariant to ensure that the view will be never harmful.

Notably, the view is dynamically updated after each reaction to a query, whereas the policy is kept unchanged once it has been statically declared by a security officer. We can rephrase this approach to dealing with the history as follows: at any point in time, the control has to confine the entailment relationship between the increasingly powerful (knowledgeable) view and the static policy.

We will illustrate this view-based approach to inference control by the following simple and straightforward example. Suppose that the policy requests to keep the propositional sentence $\varphi_{1} \wedge \varphi_{2}$ secret. Furthermore, the user is assumed to have no a priori knowledge about the instance, for which both $\varphi_{1}$ and $\varphi_{2}$ are supposed to hold, and thus $\varphi_{1} \wedge \varphi_{2}$ as well. Initially, the control generates an empty view. Then, as a first query, the user submits the sentence $\varphi_{1}$ in order to ask whether this sentence holds. The correct answer, $\varphi_{1}$, i.e., that this sentence holds, together with the empty view does not entail the single policy element, and thus the control returns the correct answer to the user in undistorted form and, accordingly, updates the view, which now comprises just the returned answer $\varphi_{1}$. Finally, as a second query, the user submits $\varphi_{2}$. Now, the correct answer, $\varphi_{2}$, together with the content of the updated view, $\varphi_{1}$, obviously entails the policy element, $\varphi_{1} \wedge \varphi_{2}$, and thus the control must suitably distort the answer. Note the dynamic "last-minute behavior" of the control: if the queries were submitted in reverse order, first $\varphi_{2}$ and then $\varphi_{1}$, then $\varphi_{2}$ would have been correctly answered and the answer to $\varphi_{1}$ would have been distorted.

In this work, we will explore an alternative approach to employ the user's history. The alternative approach aims to represent the user's history by $d y$ namically adapting the policy, thereby getting rid of the need to generate and maintain a view for the user. Intuitively, over the time, we will increasingly strengthen the policy, making it more and more restrictive as a countermeasure to the knowledge accumulated by previous answers.

To illustrate this alternative policy-adaption based approach, we reconsider the example presented above. Initially, the policy contains the sentence $\varphi_{1} \wedge \varphi_{2}$. Since the first query, $\varphi_{1}$, is harmless, the correct answer is returned to the user. Now, once one of the conjuncts occurring in the original policy element is known to the user, he must not learn the other conjunct as well. Accordingly, the control replaces the previous policy element $\varphi_{1} \wedge \varphi_{2}$ by $\varphi_{2}$ to be kept secret in future. If afterwards the second query, $\varphi_{2}$, is submitted, the control will immediately detect that the correct answer would violate the adapted policy and thus will distort the answer, as in the view-based approach.

We can also describe the policy-adaption based approach in terms of theoremproving, as sketched in the following and elaborated in more detail in the remainder of this paper. In the starting step, for each sentence contained in the declared 
policy and thus explicitly wanted to be kept secret to the user while returning answers to him, the user is supposed to aim at proving (the validity of) that sentence from the answers received. Acccordingly, for simplicity here assuming no a priori knowledge, the user initially considers every sentence contained in the declared policy to be a current proof obligation. Having received a new answer $\Phi_{i}$ in step $i$, the user can analyze all possible formal proofs for any of the current proof obligations whether and how $\Phi_{i}$ will be helpful to prove it. If the user detects such a situation, he can determine the resulting remaining proof obligations and, potentially, try to satisfy them by issuing further queries. Correspondingly, inference control can track the user's abilities, and thus control can dynamically adapt the policy by always setting it to the current set of proof obligations. In the example given above, the sole initial proof obligation is $\varphi_{1} \wedge \varphi_{2}$, which can be replaced by the new proof obligation $\varphi_{2}$, once $\varphi_{1}$ is known.

Dynamic inference control is costly, at least in general, due to the inevitable need to suitably keeping track of the history and performing some kind of theorem-proving. The basic features of policy adaption suggest the possibility of substantial improvements in computational costs at query time in comparison with the view-based approach, at least in special situations: (1) the control no longer has to maintain a separate data structure for reflecting the user's view, and (2) analyzing and remembering remaining proof obligations can bee seen as a kind of stored precomputation for the task of checking whether subsequent queries are harmful or not. Moreover, we might be able to find appropriate data structures to actually benefit from the potentials.

In the following we roughly outline such an improvement for a restricted propositional situation, where queries are just propositional atoms of the form $a_{i}$ and elements of the confidentiality policy are conjunctions of such atoms, thus of the form $a_{i_{1}} \wedge \ldots \wedge a_{i_{k}}$ with $1 \leq k$. Moreover, we will make policies redundancyfree in the sense that no policy element is a subconjunction of another policy element, just by discarding the larger one. As an example, let the policy be $\left\{a_{1} \wedge a_{2} \wedge a_{3}, a_{3} \wedge a_{4}, a_{4} \wedge a_{5}, a_{6}\right\}$, and consider the query sequence $\left\langle a_{1}, a_{2}\right\rangle$.

The current policy will be represented by a data structure that is composed of two linked parts. The "look-up part" contains all atoms still occurring in the policy, and the "reduced part" comprises the nontrivial conjunctions (having at least 2 different atoms) still to be checked. Moreover, each atom in the former part is linked to each of the conjunctions in which it occurs in the latter part. Fig. 1 shows the initial state of the data structure for the example.

If an atom $a_{i}$ is submitted as a query, the control first searches for that atom in the look-up part. If the atom is not found there, the query is censored to be harmless and correctly answered. Otherwise, there are two cases: If the atom is not linked to any nontrivial conjunction, then the atom is harmful by itself and the answer must be distorted. Otherwise, if there are links, the query is censored to be harmless and correctly answered, but the policy must be adapted by manipulating the current state of the data structure appropriately: (1) the query atom $a_{i}$ is removed from the look-up part; (2) the query atom $a_{i}$ is deleted from all the conjunctions in which it occurs; (3) if after the deletion a remaining 
initial state

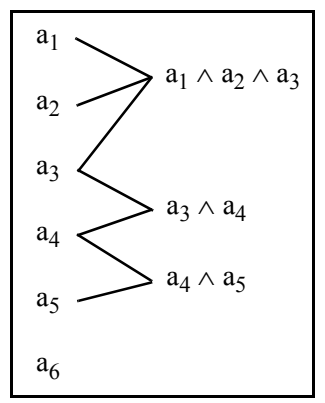

state after processing $\mathrm{a}_{1}$

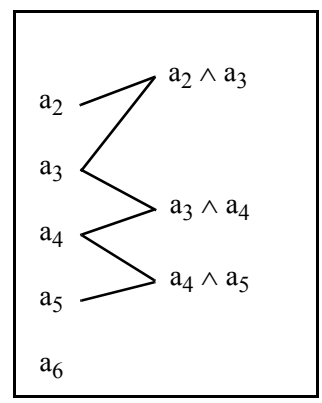

state after processing $a_{2}$

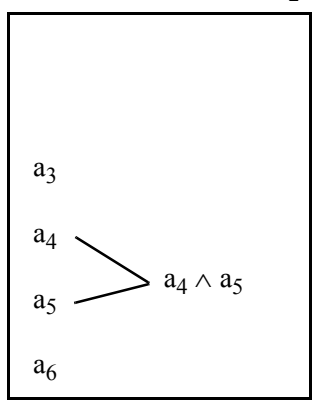

Fig. 1. Initial and subsequent states of a data structure for dynamic policy adaption

conjunction is reduced to a single atom $a_{j}$, then the conjunction is dropped at all and the corresponding link from $a_{j}$ is deleted as well; moreover, all other conjunctions in which $a_{j}$ occurs are deleted with all their links, too. Finally, if by these deletions - another atom in the look-up part has lost all its links, then that atom is deleted from the look-up part.

Fig. 11 visualizes how the control operates for the parameters specified above. Querying the atom $a_{1}$ is harmless and leads to its removal from the look-up part by (1) and its deletion from the first conjunction, which is thus reduced to $a_{2} \wedge a_{3}$ by (2). Then querying the atom $a_{2}$ is harmless again and leads to its removal from the look-up part by (1) and its deletion from the reduced conjunction, which thus becomes the single atom $a_{3}$ by (2); but this trivial conjunction is then totally dropped by (3), and the conjunction $a_{3} \wedge a_{4}$ is deleted as well.

Since only some searching and elementary link manipulations are used, the efficiency of the procedure should be evident. A full justification of the correctness is elaborated in Sect. 3 for a more general situation. Roughly summarizing, in this article we will provide the following main contributions:

- We propose the policy-adaption based approach to keeping track of the history as a promising alternative to the view-based approach (this Sect. 1).

- After introducing our basic notations, briefly reviewing the view-based approach and commenting on complexity issues (Sect.2), we fully elaborate the new approach for a special but reasonably expressive situation of Controlled Query Evaluation. This situation employs refusal as the sole distortion option and deals with a propositional information system (Sect. 33).

- We relate our approach to previous work, briefly discuss first-order information systems and evaluate the expected potentials and limitations (Sect. 4).

\section{Basic Notations and View-Based Approach}

Restricting to propositional information systems, we first introduce our basic notations. Then we briefly describe the view-based approach and state some observations on the complexity of deciding the pertinent logical implications. 


\subsection{Basic Notations}

We employ a logic-oriented approach to information systems (see, e.g., [1]), which establishes formal semantics for both query answering and updating (not considered in this paper). For simplicity, we only consider complete information systems, and we focus on propositional logic. We assume a vocabulary of propositional atoms, from which we can construct propositional sentences in the standard way, using the propositional connectives of negation and disjunction and further derived connectives. A literal is either an atom or a negated atom.

The schema (of the information system) is given by the vocabulary and the integrity constraints, which are expressed as a finite set con of sentences over this vocabulary. We consider the integrity constraints as part of any user's a priori knowledge, which in each case is given as a set of sentences over the vocabulary.

An instance $d b$ (of the information system) is a set of literals formed as follows: For each atom $\alpha$ of the vocabulary, either the atom $\alpha$ itself or the negated atom $\neg \alpha$ is an element. Given the vocabulary, it suffices to explicitly specify only those atoms that are contained in an instance (implicitly assuming for the remaining atoms that their negations are elements by default, as a kind of closed world assumption). An instance $d b$ defines a truth-value assignment to propositional atoms by making each atom $\alpha \in d b$ true and all the remaining atoms false. Such a truth-value assignment (interpretation) is inductively extended to arbitrary sentences $\Phi$ by giving the connectives the standard meaning; $\operatorname{eval}(\Phi)(d b)$ denotes the truth value assigned to $\Phi$ by $d b$. The standard notion of logical implication, or entailment, between (sets of) sentences is designated by $\models$.

As a (closed, yes/no-) query, we allow any sentence $\Phi$ of the underlying propositional logic. The correct answer to the query $\Phi$ under an instance $d b$ is given by the pertinent truth value $\operatorname{eval}(\Phi)(d b)$; however, for convenience, we alternatively express the correct answer by $e^{2} a l^{*}(\Phi)(d b)$ that denotes either $\Phi$ or $\neg \Phi$ in a straightforward way. We aim at controlling any sequence of queries $Q:=\left\langle\Phi_{1}, \Phi_{2}, \ldots, \Phi_{i}, \ldots, \Phi_{k}\right\rangle$ where the query $\Phi_{i}$ is submitted by some user at the point in time $i$; for simplicity of the presentation, we focus on only one user.

While the user is granted a general access right for reading (querying), a security officer declares a confidentiality policy as a finite set psec of propositional sentences, called potential secrets, in order to confine the actual information gain that can be achieved by the user. Here the qualification "potential" indicates that these sentences are not necessarily true in the actual instance. Following the principle of open design, the user is supposed to be aware of this declaration, as well as of all other features of the control mechanism. In order to prevent the user from ever inferring that any sentence $\Psi \in$ psec actually holds, we follow the refusal approach to inference control [1165], i.e., if an informative answer to a query would be harmful, then the control reacts by returning a special symbol mum. In general, the refusal approach has to examine not only whether the correct answer to a query is harmful but also whether its negation would be harmful, in order to prevent so-called meta-inferences.

Besides the policy, in general the control mechanism also has to consider the (postulated) a priori knowledge of the user and the answers to previously issued 
queries. To do so, the control might maintain a user log. Basically, such a user $\log$ then just contains a set $\log$ of propositional sentences. In principle, both the policy and the user log might be updated while processing queries; the current versions of them taken together form the current state $s_{i}:=\left(\right.$ psec $\left._{i}, \log _{i}\right)$ of the control mechanism. The initial state is obtained by setting $\operatorname{psec}_{0}:=$ psec and $\log _{0}:=$ prior, where prior can be any suitable superset of the constraints con not being in conflict with psec, i.e., prior $\forall \neq \Psi$ for all $\Psi \in$ psec. In this work, for simplicity, we will not elaborate the treatment of the a priori knowledge prior in depth: we just leave it empty in our examples, and we simply process it like a sequence of queries within our initialization subprotocol.

Definition 1 (controlled query evaluation). Let be given an instance $d b$, a finite set $\log _{i-1}$ of sentences (for explicitly reflecting the assumed user's current knowledge about the instance), and a finite set psec $_{i-1}$ of sentences (for representing the current version of the confidentiality policy). Then a function cqe $\left(d b\right.$, psec $\left._{i-1}, \log _{i-1}, \Phi_{i}\right)$ defines a controlled query evaluation of a query $\Phi_{i}$ by generating a triple $\left(\right.$ ans $_{i}$, psec $\left._{i}, \log _{i}\right)$, where ans $s_{i}$ is the answer returned to the user, and psec $_{i}$ and $\log _{i}$ together form the updated state.

Furthermore, for the initializations specified above, this function is inductively extended to any query sequence $Q:=\left\langle\Phi_{1}, \ldots, \Phi_{i}, \ldots, \Phi_{k}\right\rangle$ by applying it stepwise in a straightforward way:

$$
\begin{aligned}
& \operatorname{cqe}\left(d b, \operatorname{psec}_{0}, \log _{0}, Q\right):= \\
& \left\langle\left(\text { ans }_{1}, \operatorname{psec}_{1}, \log _{1}\right), \ldots,\left(\text { ans }_{i}, \text { psec }_{i}, \log _{i}\right), \ldots,\left(\text { ans }_{k}, \operatorname{psec}_{k},, \log _{k}\right)\right\rangle
\end{aligned}
$$

We are now ready to present our formal definition of the confidentiality requirement we want to achieve by a controlled query evaluation. Roughly summarized, given a potential secret $\Psi$ declared in the (original) policy psec, this requirement is expressed in terms of the indistinguishability - from the point of view of the user - of the actual instance $d b$ from an alternative instance $d b^{s}$ that does not satisfy the potential secret considered.

Definition 2 (confidentiality). A controlled query evaluation cqe preserves confidentiality iff

for all instances $d b$,

for all finite sets of sentences psec (original confidentiality policy),

for all finite sets of sentences prior (a priori knowledge)

satisfied by $d b$ and such that prior $\forall \forall \Psi$ for all $\Psi \in$ psec,

for all query sequences $Q$, and

for all potential secrets $\Psi \in$ psec

there exists an alternative instance $d b^{s}$ satisfying prior such that:

1. [indistinguishability]:

$$
\text { cqe }(d b, \text { psec, prior }, Q)=c q e\left(d b^{s}, \text { psec, } \text { prior }, Q\right)
$$

2. [possibility of false potential secrets]:

$$
e^{e v a l}{ }^{*}(\Psi)\left(d b^{s}\right)=\neg \Psi
$$




\section{$2.2 \quad$ View-Based Approach}

The view-based approach to Controlled Query Evaluation, as surveyed in [5], keeps track of the history by only updating the user log, while leaving the original policy unchanged. For the specific setting described above, i.e., refusal under known potential secrets for a propositional information system dealing with closed (yes/no-)queries, the function $c q e^{v i e w}$ is defined by its outputs as follows:

$$
\begin{aligned}
\text { ans }_{i}:= & \text { if } \log _{i-1} \models \operatorname{eval}^{*}\left(\Phi_{i}\right)(d b) \text { then } \text { eval }^{*}\left(\Phi_{i}\right)(d b) \text { else } \\
& \text { if }(\text { exists } \Psi)(\Psi \in \text { psec and } \\
& \left.\quad\left(\log _{i-1} \cup\left\{\Phi_{i}\right\} \models \Psi \text { or } \log _{i-1} \cup\left\{\neg \Phi_{i}\right\} \models \Psi\right)\right) \\
& \text { then mum else eval }\left(\Phi_{i}\right)(d b) \\
\text { psec }_{i}:= & \text { psec } \\
\log _{i}:= & \text { if } \text { ans }_{i}=\text { mum then } \log _{i-1} \text { else } \log _{i-1} \cup\left\{\text { ans }_{i}\right\}
\end{aligned}
$$

Proposition 1 ([6]). The function cqe view preserves confidentiality in the sense of Def. Q2.

Definition (4) of the controlled answer indicates that the task of inference control is closely related to the problem of deciding on logical implications of the form $\chi \models \Psi$, where the finite set of sentences $\chi$ - equivalently identified with the corresponding sentence formed as the conjunction over this set - denotes some potential knowledge of the user and $\Psi$ is a policy element. This decision problem is well-known to be of high computational complexity in general, and thus we can expect to control answers efficiently only under some restrictions of the expressiveness of the languages for the sentences $\chi$ and $\Psi$, respectively.

As a starting point, we first observe the following: If both $\chi$ and $\Psi$ are already specified in disjunctive normal form for a finite vocabulary, i.e., as a disjunction of so-called minterms that are built as a conjunction of literals (atoms or negated atoms) ranging over all atoms in the vocabulary, then $\chi \models \Psi$ holds if and only if each minterm of $\chi$ is also a minterm of $\Psi$.

For a slightly relaxed situation where both $\chi$ and $\Psi$ are specified as a disjunctive form, i.e., a disjunction of conjunctions of literals ranging over different atoms in the vocabulary, the sufficiency part of this observation can be generalized along the following lines of reasoning, often referred to as subsumption. First, if some disjunctive sentences $\eta_{1}$ and $\eta_{2}$ are (syntactically) related such that each disjunct of $\eta_{1}$ is also a disjunct of $\eta_{2}$ - or at least (semantically) implies some disjunct of $\eta_{2}-$, then the (semantic) implication $\eta_{1} \models \eta_{2}$ holds, since $\eta_{2}$ is an obvious weakening of $\eta_{1}$. Dually, if some conjunctive sentences $\theta_{1}$ and $\theta_{2}$ are (syntactically) related such that each conjunct of $\theta_{1}$ is also a conjunct of $\theta_{2}-$ or is at least (semantically) implied by some conjunct of $\theta_{2}-$, then the (semantic) implication $\theta_{2} \models \theta_{1}$ holds, since $\theta_{2}$ is an obvious strengthening of $\theta_{1}$.

Unfortunately, the necessity part of the observation stated above cannot be generalized for arbitrary disjunctive forms. However, the necessity part holds indeed, if the sentence $\Psi$ consists of all the prime implicants of $\Psi$, i.e., (1) each disjunct of $\Psi$ is minimal in the sense that discarding any of the literals in the 
conjunction that constitutes this disjunct would result in a non-equivalent sentence, and (2) $\Psi$ contains all minimal disjuncts (conjunctions of literals ranging over different atoms in the vocabulary) that imply $\Psi$.

Proposition 2. Let $\chi$ be a disjunctive form and $\Psi$ a disjunctive form that consists of all its prime implicants. Then $\chi \models \Psi$ holds if and only if for each disjunct of $\chi$ there is a disjunct of $\Psi$ such that each literal occurring as a conjunct of the latter disjunct also appears as a conjunct of the former disjunct.

\section{Policy Adaption for Propositional Information Systems}

We now present our new concept of the policy-adaption based approach in detail, exhibit an appropriate data structure for representing the current policy, and then demonstrate the correctness and comment on the efficiency.

\subsection{Outline and Examples}

To elaborate the policy-adaption based approach, we aim at defining the corresponding function $c q e^{p o l}$ for controlled query evaluation such that the following properties (further explained below) hold:

1. The parameter $\log$ could be dropped.

2. The history is reflected in the current version $p s e c_{i}$ of the policy.

3. The generated outputs ans $_{i}$ are the same as for $c q e^{\text {view }}$.

4. The current version $p s e c_{i}$ is converted to be redundancy-free (see below).

5. The current version $\operatorname{psec}_{i}$ is converted to be fully vulnerable (see below).

We first outline the basic techniques to achieve these properties, then exemplify these techniques, and finally present and verify a comprehensive algorithm for $c q e^{p o l}$ leading to a controlled query evaluation based on these techniques.

By property 3 and as a corollary to the result for $c q e^{\text {view }}$ stated in Prop. 1 , the function $c q e^{p o l}$ will preserve confidentiality in the sense of Def. 2 as well.

Regarding property 4, demanding the policy to be redundancy-free, we can observe the following by inspecting the guarding condition in the second line and the third line of (4): If a policy psec contains two different potential secrets $\Psi_{1}$ and $\Psi_{2}$ such that $\Psi_{1} \models \Psi_{2}$, then we can remove $\Psi_{1}$ from the policy without affecting the answer. For, if a user knowledge $\log \cup\{\Phi\}$ or $\log \cup\{\neg \Phi\}$, respectively, implies $\Psi_{1}$, then that knowledge also implies $\Psi_{2}$; thus the outcome of the guarding condition remains the same after removing $\Psi_{1}$. Accordingly, we will keep the set psec redundancy-free in the sense that none of its elements implies another one.

Regarding property 5, demanding the policy to be fully vulnerable, we further observe the following: If a policy psec contains a potential secret $\Psi$ such that $\log \models \neg \Psi$ holds for the current user knowledge $\log$, then we can remove $\Psi$ from the policy. For, by monotonicity, this property will always be preserved later on and thus the confidentiality requirement expressed by $\Psi$ will never be hurt. 
Regarding the properties 2 and 3, which demand an appropriate reflection of the history in psec $_{i}$ such that the same outputs are generated as in the viewbased approach, again by inspecting the guarding condition in the second line and the third line of (4), we have to inductively achieve an equivalence of the following kind (to be made more precise later on), where $\Delta_{i}$ denotes the query $\Phi_{i}$ or its negation $\neg \Phi_{i}$, respectively:

$$
\begin{aligned}
& (\text { exists } \Psi)\left(\Psi \in \text { psec and } \log _{i-1} \cup\left\{\Delta_{i}\right\} \models \Psi\right) \text { iff } \\
& (\text { exists } \Psi)\left(\Psi \in \text { psec }_{i-1} \text { and }\left\{\Delta_{i}\right\} \models \Psi\right)
\end{aligned}
$$

To attain such a goal, we first impose all queries $\Phi_{i}$ and all policy elements $\Psi$ to be given as a disjunctive form. Moreover, we additionally extend each policy element such that it contains all its prime implicants in order to profit from the efficiently verifiable characteristic property of $\chi \models \Psi$ given in Prop. 2.

Next, again for easily exploiting that property, in general we aim at representing a policy element of the form $\Psi=\Psi_{1} \vee \ldots \vee \Psi_{m}$ that constitutes a nontrivial disjunction with $2 \leq m$ as the set of its disjuncts $\left\{\Psi_{1}, \ldots, \Psi_{m}\right\}$. To achieve a homogeneous treatment with a policy element of the form $\Psi=\Psi_{1}$ having only one disjunct, we then have to represent such an element as the singleton set $\left\{\Psi_{1}\right\}$. The set representations introduced will not affect the wanted equivalence, since they are functional equivalent with the original forms. If there are no semantic ambiguities, i.e., from a special context under consideration it is clear whether two disjuncts (implicants) belong to the same policy element or not, as in the examples below, we will omit the set notation for the sake of readibility.

Finally, to deal with disjunctive answers of the form $\Phi=\Phi_{1} \vee \ldots \vee \Phi_{n}$ with $2 \leq n$, we will introduce policy branches: for each disjunct $\Phi_{l}$, a copy of the current policy is generated and then inspected regarding implications that result from $\Phi_{l}$ alone. Subsequently, each of these branches has to be maintained with reference to the pertinent $\Phi_{l}$ until a definite answer that $\neg \Phi_{l}$ holds is given; then the branch is obviously contradictory and thus must be removed.

Example 1. Consider the following situation:

$d b:=\left\{a_{1}, \neg a_{2}, \neg a_{3}, a_{4}\right\}$ is the instance,

$Q:=\left\langle a_{1}, a_{2}, a_{3}, a_{4}\right\rangle$ is the query sequence,

psec $:=\left\{\neg a_{1} \wedge \neg a_{2} \wedge a_{3} \wedge \neg a_{4}, a_{1} \wedge \neg a_{2} \wedge \neg a_{3} \wedge a_{4}\right\}$ is the policy, and

$\log _{0}:=\emptyset$ is the void a priori knowledge.

Then $\left\langle a_{1}, \neg a_{2}, \neg a_{3}, a_{4}\right\rangle$ is the correct answer sequence, and the instance defines the first potential secret to be false and the second one to be true.

Controlling the first query $a_{1}$, we see that neither $a_{1}$ nor $\neg a_{1}$ implies any of the potential secrets, and thus the correct answer $a_{1}$ can be returned, and it would be inserted into the user log by the view-based approach such that we would have $\log _{1}:=\left\{a_{1}\right\}$. Since the first potential secret is no longer vulnerable, we can remove it from the policy. Furthermore, once the user knows $a_{1}$, we now have to protect the remainder of the second potential secret, i.e., we can drop $a_{1}$ from $a_{1} \wedge \neg a_{2} \wedge \neg a_{3} \wedge a_{4}$. Thus we get

$$
\text { psec }_{1}:=\left\{\neg a_{2} \wedge \neg a_{3} \wedge a_{4}\right\} \text {. }
$$




\begin{tabular}{|c|c|c|}
\hline Description and Branch & \begin{tabular}{|l} 
Disjuncts (given \\
prime implicants)
\end{tabular} & $\begin{array}{l}\text { Disjuncts (additional } \\
\text { prime implicants) }\end{array}$ \\
\hline $\begin{array}{l}\text { sole element } \\
\text { (original) }\end{array}$ & $\mid \begin{array}{l}a_{1} \wedge \neg a_{3} \wedge \neg a_{4} \\
a_{1} \wedge \neg a_{2} \wedge a_{4} \\
a_{2} \wedge a_{3} \wedge a_{4} \\
\neg a_{1} \wedge a_{2} \wedge \neg a_{3} \\
\end{array}$ & $\begin{array}{l}a_{2} \wedge \neg a_{3} \wedge \neg a_{4} \\
a_{1} \wedge \neg a_{2} \wedge \neg a_{3} \\
a_{1} \wedge a_{3} \wedge a_{4} \\
\neg a_{1} \wedge a_{2} \wedge a_{4} \\
\end{array}$ \\
\hline $\begin{array}{l}\text { sole element } \\
\left(\text { after answer } a_{1}\right)\end{array}$ & $\mid \begin{array}{l}\neg a_{3} \wedge \neg a_{4} \\
\neg a_{2} \wedge a_{4} \\
a_{2} \wedge a_{3} \wedge a_{4} \\
(\text { complementary) }\end{array}$ & $\begin{array}{l}a_{2} \wedge \neg a_{3} \wedge \neg a_{4} \\
\neg a_{2} \wedge \neg a_{3} \\
a_{3} \wedge a_{4} \\
(\text { complementary) }\end{array}$ \\
\hline $\begin{array}{l}\text { sole element } \\
\left(\text { after answer } a_{1}\right. \\
\text { and subsumption) }\end{array}$ & \begin{tabular}{|l}
$\neg a_{3} \wedge \neg a_{4}$ \\
$\neg a_{2} \wedge a_{4}$ \\
$($ subsumed) \\
$($ complementary)
\end{tabular} & $\begin{array}{l}\text { (subsumed) } \\
\neg a_{2} \wedge \neg a_{3} \\
a_{3} \wedge a_{4} \\
\text { (complementary) }\end{array}$ \\
\hline $\begin{array}{l}\text { sole element } \\
\left.\text { (after answers } a_{1}, \neg a_{2} \vee a_{3}\right) \\
\text { for branch }\left\{\neg a_{2}\right\}\end{array}$ & $\mid \begin{array}{l}\neg a_{3} \wedge \neg a_{4} \\
a_{4} \\
\text { (subsumed) } \\
(\text { complementary) }\end{array}$ & $\begin{array}{l}\text { (subsumed) } \\
\neg a_{3} \\
a_{3} \wedge a_{4} \\
\text { (complementary) }\end{array}$ \\
\hline $\begin{array}{l}\text { sole element } \\
\left.\text { (after answers } a_{1}, \neg a_{2} \vee a_{3}\right) \\
\text { for branch }\left\{a_{3}\right\}\end{array}$ & $\mid$\begin{tabular}{||l}
$($ complementary) \\
$\neg a_{2} \wedge a_{4}$ \\
(subsumed) \\
(complementary)
\end{tabular} & $\begin{array}{l}\text { (subsumed) } \\
\text { (complementary) } \\
a_{4} \\
(\text { complementary })\end{array}$ \\
\hline $\begin{array}{l}\text { sole element } \\
\text { (after answers } a_{1}, \neg a_{2} \vee a_{3} \\
\text { and subsumption) } \\
\text { for branch }\left\{\neg a_{2}\right\}\end{array}$ & \begin{tabular}{||l}
$($ subsumed $)$ \\
$a_{4}$ \\
$($ subsumed $)$ \\
(complementary)
\end{tabular} & $\begin{array}{l}\text { (subsumed) } \\
\neg a_{3} \\
\text { (subsumed) } \\
\text { (complementary) }\end{array}$ \\
\hline $\begin{array}{l}\text { sole element } \\
\text { (after answers } a_{1}, \neg a_{2} \vee a_{3} \\
\text { and subsumption) } \\
\text { for branch }\left\{a_{3}\right\}\end{array}$ & $\mid$\begin{tabular}{||l} 
(complementary) \\
$($ subsumed $)$ \\
(subsumed) \\
(complementary)
\end{tabular} & $\begin{array}{l}\text { (subsumed) } \\
\text { (complementary) } \\
a_{4} \\
\text { (complementary) }\end{array}$ \\
\hline
\end{tabular}

Fig. 2. A converted and then stepwise adapted confidentiality policy

Similarly, stepwise controlling the second query $a_{2}$ and the third query $a_{3}$, we (would) get the following:

$$
\begin{array}{ll}
\log _{2}:=\left\{a_{1}, \neg a_{2}\right\}, & \operatorname{psec}_{2}:=\left\{\neg a_{3} \wedge a_{4}\right\}, \\
\log _{3}:=\left\{a_{1}, \neg a_{2}, \neg a_{3}\right\}, & \operatorname{psec}_{3}:=\left\{a_{4}\right\} .
\end{array}
$$

Finally, controlling the fourth query $a_{4}$, we immediately see that the correct answer violates the policy, and thus the answer must be refused. Notably, if the correct answer was $\neg a_{4}$, then that answer would have to be refused as well.

Example 2. Consider the following situation, the processing of which is further illustrated in Fig. 2,

$d b:=\left\{a_{1}, \neg a_{2}, \neg a_{3}, a_{4}\right\}$ is the instance, the same as before,

$Q:=\left\langle a_{1}, \neg a_{2} \vee a_{3}, a_{3}, a_{4}\right\rangle$ is the query sequence,

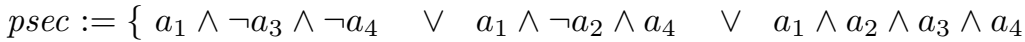

$\left.\vee \neg a_{1} \wedge a_{2} \wedge a_{3} \wedge a_{4} \vee \neg \neg a_{1} \wedge a_{2} \wedge \neg a_{3}\right\}$ is the policy, and

$\log _{0}:=\emptyset$ is the void a priori knowledge.

Obviously, then $\left\langle a_{1}, \neg a_{2} \vee a_{3}, \neg a_{3}, a_{4}\right\rangle$ is the correct answer sequence, and the instance defines the sole potential secret to be true. 
At initialization time, we observe that the sole policy element can be equivalently simplified by combining the two disjuncts $a_{1} \wedge a_{2} \wedge a_{3} \wedge a_{4}$ and $\neg a_{1} \wedge a_{2} \wedge a_{3} \wedge a_{4}$ into the prime implicant $a_{2} \wedge a_{3} \wedge a_{4}$. Furthermore, even afterwards the policy element does not contain all its prime implicants; in fact, we have to add four further prime implicants, namely

$a_{2} \wedge \neg a_{3} \wedge \neg a_{4}, a_{1} \wedge \neg a_{2} \wedge \neg a_{3}, a_{1} \wedge a_{3} \wedge a_{4}$, and $\neg a_{1} \wedge a_{2} \wedge a_{4}$.

Subsequently, we replace the sole policy element by the set of its disjuncts (prime implicants) and get the following representation of the policy:

$$
\begin{aligned}
& \text { psec }_{0}:=\left\{a_{1} \wedge \neg a_{3} \wedge \neg a_{4}, a_{1} \wedge \neg a_{2} \wedge a_{4}, a_{2} \wedge a_{3} \wedge a_{4}, \neg a_{1} \wedge a_{2} \wedge \neg a_{3},\right. \\
& \left.a_{2} \wedge \neg a_{3} \wedge \neg a_{4}, \quad a_{1} \wedge \neg a_{2} \wedge \neg a_{3}, \quad a_{1} \wedge a_{3} \wedge a_{4}, \neg a_{1} \wedge a_{2} \wedge a_{4}\right\} .
\end{aligned}
$$

Controlling the first query $a_{1}$, we see that neither $a_{1}$ nor $\neg a_{1}$ implies any of the potential secrets, and thus the correct answer $a_{1}$ can be returned. Since the policy elements containing the complementary literal $\neg a_{1}$ are no longer vulnerable, we can remove them from the policy. Furthermore, once the user knows $a_{1}$, we can drop $a_{1}$ from the remaining elements. Additionally, we can remove elements that have become redundant, which is equivalent to being subsumed by a shorter disjunct. Altogether we get

$$
\text { psec }_{1}:=\left\{\neg a_{3} \wedge \neg a_{4}, \neg a_{2} \wedge a_{4}, \neg a_{2} \wedge \neg a_{3}, a_{3} \wedge a_{4}\right\} .
$$

Controlling the second query $\neg a_{2} \vee a_{3}$, we see again that neither the positive answer $\neg a_{2} \vee a_{3}$ nor the negative answer $a_{2} \wedge \neg a_{3}$ implies any of the potential secrets, and thus the correct answer can be returned. However, since the correct answer is a disjunction, we split the policy into branches, one for the case that $\neg a_{2}$ is actually true and another one for the case that $a_{3}$ is actually true.

In the branch for $\neg a_{2}$, we can drop the occurrences of $\neg a_{2}$ from two of the elements, yielding the reduced elements $a_{4}$ and $\neg a_{3}$. As there are no occurrences of the complementary literal $a_{2}$, all elements are still vulnerable. Additionally, however, we can remove the then subsumed elements $\neg a_{3} \wedge \neg a_{4}$ and $a_{3} \wedge a_{4}$. Thus we get

$$
\text { psec }_{2}\left[\neg a_{2}\right]:=\left\{a_{4}, \neg a_{3}\right\} .
$$

In the branch for $a_{3}$, we can drop the occurrence of $a_{3}$ from one of the elements, yielding the reduced element $a_{4}$, and we can remove the elements $\neg a_{3} \wedge \neg a_{4}$ and $\neg a_{2} \wedge \neg a_{3}$, in which the complementary literal $\neg a_{3}$ occurs. Additionally, the element $\neg a_{2} \wedge a_{4}$ is now subsumed and thus can be removed. Thus we get

$$
\text { psec }_{2}\left[a_{3}\right]:=\left\{a_{4}\right\} \text {. }
$$

Controlling the third query $a_{3}$, we see that the correct answer $\neg a_{3}$ makes the policy branch for $a_{3}$ contradictory and implies an element in the remaining branch for $\neg a_{2}$. Accordingly, the answer must be refused and both policy branches remain unchanged. Finally, controlling the fourth query $a_{4}$, we see that the correct answer $a_{4}$ implies a policy element in both branches, and thus the answer must be refused as well.

\subsection{Protocol for Policy Adaption and Correctness}

Having introduced the basic techniques, we are now ready to specify the types, inputs and methods of our new approach of policy adaption more formally. 


\section{Protocol for Policy Adaption.}

\section{types.}

$\mathscr{L}$ propositional sentences;

$\mathscr{L}_{\mathrm{df}} \subseteq \mathscr{L}$ propositional sentences in disjunctive form;

$\mathscr{L}_{\text {pi }} \subseteq \mathscr{L}_{\mathrm{df}}$ propositional sentences that consist of all their prime implicants;

$\mathscr{L}_{\text {li }} \subseteq \mathscr{L}_{\mathrm{pi}}$ literals;

$\mathscr{L}_{\text {im }} \subseteq \mathscr{L}_{\mathrm{df}}$ implicants (conjunctions of literals over distinct atoms);

$\mathscr{C} \subseteq$ finite $\wp \mathscr{L}$ declared confidentiality policies;

$\mathscr{M} \subseteq$ finite $\wp \wp \mathscr{L}_{\text {im }}$ converted confidentiality policies

as multisets of "identified policy elements";

$\mathscr{B} \subseteq$ finite $\mathscr{M} \times \wp \mathscr{L}_{\text {li }} \quad$ policy branches; //written as imsets [liset $]$;

$\mathscr{Q} \subseteq \mathscr{L}$ queries.

\section{subprotocol: initialization.}

input: psec : $\mathscr{C}$;

prior : $\wp \mathscr{L}$

method:

1. $\sec _{\emptyset}:=$ psec;

2. modify $\sec _{\emptyset}$ as follows:

foreach $\Psi \in \sec _{\emptyset}$ do

convert $\Psi$ such that it becomes the disjunction of all its prime implicants;

foreach $\Psi \in \sec _{\emptyset}$ do

replace $\Psi$ having form $\Psi_{1} \vee \ldots \vee \Psi_{m}$ by the representing set $\left\{\Psi_{1}, \ldots, \Psi_{m}\right\}_{\Psi}$;

3. psecb $_{0}:=\left\{\sec _{\emptyset}[\emptyset]\right\} ; / /$ only one policy branch of form $\{\{\ldots\}, \ldots,\{\ldots\}\}[\emptyset]$

4. process prior like a sequence of queries. //not elaborated for lack of space

\section{subprotocol: generation (of answer and policy).}

input: $\Phi_{i}: \mathscr{L}$;

method:

$$
\operatorname{psecb}_{i-1}: \mathscr{B}
$$

1. convert $\Phi_{i}$ into disjunctive form $\Phi_{i, 1} \vee \ldots \vee \Phi_{i, n}$;

2. ans $_{i}:=$ if $\Phi_{i}$ violates secb $_{i-1}$ or $\neg \Phi_{i}$ violates psecb $_{i-1}$

then mum

else eval $^{*}\left(\Phi_{i}\right)(d b)$;

3 . if ans $_{i}=\Phi_{i}\left(\right.$ let $\left.\Phi_{i}=\Phi_{i, 1} \vee \ldots \vee \Phi_{i, n}\right)$

then $\operatorname{psecb}_{i}:=\emptyset$;

foreach disjunct $\Phi_{i, j}$ of $\Phi_{i}$ do

lit $_{j}:=\left\{\varphi \mid \varphi\right.$ occurs in $\left.\Phi_{i, j}\right\}$;

copy $_{j}:=\left\{\sec \left[D \cup \operatorname{lit}_{j}\right] \mid \sec [D] \in\right.$ psecb $\left._{i-1}\right\}$;

foreach literal $\varphi$ of $\Phi_{i, j}$ do perform policy adaption for $\varphi$ and $c_{0 p} y_{j}$; psecb $_{i}:=$ psecb $_{i} \cup \operatorname{copy}_{j}$

elseif ans $_{i}=\neg \Phi_{i}\left(\right.$ let $\left.\neg \Phi_{i}=\neg \Phi_{i, 1} \wedge \ldots \wedge \neg \Phi_{i, n}\right)$

then $\operatorname{psecb}_{i}:=\operatorname{psec}_{i-1}$;

foreach conjunct $\neg \Phi_{i, j}$ of $\neg \Phi_{i}\left(\right.$ let $\left.\neg \Phi_{i, j}=\varphi_{1} \vee \ldots \vee \varphi_{k}\right)$ do

copy $:=\emptyset$;

foreach literal $\varphi_{l}$ of $\neg \Phi_{i, j}$ do

copy $_{l}:=\left\{\sec \left[D \cup\left\{\varphi_{l}\right\}\right] \mid \sec [D] \in \operatorname{psec}_{i}\right\} ;$

perform policy adaption for $\varphi_{l}$ and $\operatorname{cop}_{l}$;

copy $:=$ copy $\cup$ copyl;

psecb $_{i}:=$ copy. 


\section{subprotocol: violation (test).}

input: $\Phi: \mathscr{L}$

method:

psecb : $\mathscr{B}$;

convert $\Phi$ into disjunctive form;

$/ /$ nothing to do if $\Phi=\Phi_{i}$, i.e., violation test is performed for current query

if there exists a branch $\sec [D]$ of policy psecb and

there exists a disjunct $\Phi_{j}$ of (negated) query $\Phi$

such that $\Phi_{j} \wedge \bigwedge_{\varphi \in D} \varphi$ is not contradictory

$/ /$ guaranteed if $\Phi=\operatorname{eval}^{*}\left(\Phi_{i}\right)(d b)$

and

there exists $\{\ldots\}_{\tilde{\Psi}} \in \sec _{\emptyset}$ such that $/ / \tilde{\Psi}$ "uniformly identifies" a policy element for all branches $\sec [D]$ of policy psecb and

for all disjuncts $\Phi_{j}$ of (negated) query $\Phi$

such that $\Phi_{j} \wedge \bigwedge_{\varphi \in D} \varphi$ is not contradictory

there exists a disjunct $\tilde{\Psi}_{r} \in\{\ldots\}_{\tilde{\Psi}} \in \sec$ such that $\Phi_{j} \models \tilde{\Psi}_{r}$ (by subsumption)

then return true (violation)

else return false (no violation).

\section{subprotocol: adaption (for literal and policy copy).}

input: $\varphi: \mathscr{L}_{1 \mathrm{i}}$;

var copy : $\mathscr{B} ; / /$ copy is used as input-and-output parameter

method: // modify copy as follows

foreach policy branch $\sec _{j}\left[D_{j}\right] \in$ copy do

1. if $\neg \varphi \in D_{j}$

then delete branch $\sec _{j}\left[D_{j}\right]$

else foreach $\left\{\chi_{1}, \ldots, \chi_{r}\right\}_{\Psi} \in \sec _{j}$ do

foreach $\chi \in\left\{\chi_{1}, \ldots, \chi_{r}\right\}_{\Psi}$ do

if $\varphi$ occurs in $\chi$ then $\operatorname{drop} \varphi$ from $\chi$;

if $\neg \varphi$ occurs in $\chi$ then remove $\chi$ from $\left\{\chi_{1}, \ldots, \chi_{r}\right\}_{\Psi}$;

foreach distinct $\chi_{1}, \chi_{2} \in\left\{\chi_{1}, \ldots, \chi_{r}\right\}_{\Psi}$ do

if $\chi_{1} \models \chi_{2}$ (by subsumption) then remove $\chi_{1}$ from $\left\{\chi_{1}, \ldots, \chi_{r}\right\}_{\Psi}$;

2. foreach $\left\{\chi_{1}, \ldots, \chi_{r}\right\}_{\Psi},\left\{\bar{\chi}_{1}, \ldots, \bar{\chi}_{\bar{r}}\right\}_{\bar{\Psi}} \in \sec _{j}$ with $\Psi \neq \bar{\Psi}$ do

if $\chi_{1} \vee \ldots \vee \chi_{r} \models \bar{\chi}_{1} \vee \ldots \vee \bar{\chi}_{\bar{r}}$

then replace $\left\{\chi_{1}, \ldots, \chi_{r}\right\}_{\Psi}$ by $\emptyset_{\Psi} / /$ consider $\emptyset_{\Psi}$ as removed.

As explained in Sect. 3.1 the protocol for policy adaption has been designed to achieve the same effects as the view-based approach. Thus the protocol is claimed to be correct with respect to the view-based approach and, accordingly by Prop. 1, to preserve confidentiality. The latter claim is stated in the following theorem, the proof of which justifies the former claim.

Theorem 1. The function cqe $e^{\text {pol }}$ as defined by the Protocol for Policy Adaption preserves confidentiality in the sense of Def. Q.

Proof. For lack of space, we only outline the inductive proof, which follows the informal arguments presented in Sect. 3.1. Basically, the induction will deal with the following items and notations: 
- hist $_{i-1}:=\bigvee_{k} \bigwedge_{l} \beta_{k, l}$ equivalently represents the user $\log \log _{i-1}$ under the view-based approach as a single sentence converted into disjunctive form.

$-\Delta_{i}:=\bigvee_{k^{\prime \prime}} \chi_{k^{\prime \prime}}$ in disjunctive form denotes the query $\Phi_{i}$ or its negation $\neg \Phi_{i}$.

- tent $_{i}:=$ hist $_{i-1} \wedge \Delta_{i}=\bigvee_{k, k^{\prime \prime}}\left(\bigwedge_{l} \beta_{k, l} \wedge \chi_{k^{\prime \prime}}\right)$ then represents a left-hand side in a violation test according to (4), but so far ignoring that contradictory disjuncts might occur.

- tent $t_{i}^{r e d}:=\bigvee_{\bar{k}, \bar{k}^{\prime \prime}}\left(\bigwedge_{l} \beta_{\bar{k}, l} \wedge \chi_{\bar{k}^{\prime \prime}}\right)$ in disjunctive form results from tent ${ }_{i}$ by discarding all contradictory disjuncts (containing both an atom $\alpha$ and the negated literal $\neg \alpha$ ). The special case that tent $t_{i}^{\text {red }}$ becomes the empty disjunction only happens if $\log _{i-1} \models e v a l^{*}\left(\Phi_{i}\right)(d b)$ and $\Delta_{i}=\neg e v a l^{*}\left(\Phi_{i}\right)(d b)$.

- $\mathscr{D}_{i-1}$ is the set of tags $D$ occurring in the current policy psecb $_{i-1}$.

- $\operatorname{psecb}_{i-1}:=\left\{\sec _{D}[D] \mid D \in \mathscr{D}_{i-1}\right\}$ then describes the elements of that policy.

One can verify that the generation subprotocol establishes a one-to-one correspondance between the set of non-contradictory disjuncts $\bigwedge_{l} \beta_{k, l}$ of hist ${ }_{i-1}$, ranging over all pertinent $k$, and $\mathscr{D}_{i-1}$, such that for each $k$ the corresponding $\operatorname{tag} D$ satisfies $D=\left\{\beta \mid \beta=\beta_{k, l}\right.$ for some $\left.l\right\}$. Note that if the generation subprotocol tentatively forms a branch corresponding to a contradictory disjunct, then this fact is detected by performing the adaption subprotocol, which leads to an immediate deletion of that branch.

Then we assert and comment the equivalence of the following assertions:

1. (exists $\Psi)\left(\Psi \in\right.$ psec and $\left.\log _{i-1} \cup\left\{\Delta_{i}\right\} \models \Psi\right)$.

Such a kind of assertion is checked by the view-based approach according to (4), to be shown to satisfy the equivalence given by "(77) iff (8)".

2. (exists $\Psi)\left(\Psi \in\right.$ psec and tent $\left.i_{i}^{\text {red }}=\Psi\right)$.

The set on the left-hand side of $=$ is represented as a single sentence, which is formed as the conjunction over all elements of that set and then converted into disjunctive form (with discarding of contradictory disjuncts).

3. $\left(\operatorname{exists} \tilde{\Psi}_{s}\right)\left(\tilde{\Psi}_{s}=\left\{\tilde{\Psi}_{s, 1}, \ldots, \tilde{\Psi}_{s, m}\right\} \in \sec _{\emptyset}\right.$ and $\left.\bigvee_{\bar{k}, \bar{k}^{\prime \prime}}\left(\bigwedge_{l} \beta_{\bar{k}, l} \wedge \chi_{\bar{k}^{\prime \prime}}\right) \models \bigvee_{r} \tilde{\Psi}_{s, r}\right)$. Here $\left\{\tilde{\Psi}_{s, 1}, \ldots, \tilde{\Psi}_{s, m}\right\}$ are the initially determined prime implicants of $\tilde{\Psi}_{s}$.

4. (exists $\left.\tilde{\Psi}_{s}\right)\left(\tilde{\Psi}_{s}=\left\{\tilde{\Psi}_{s, 1}, \ldots, \tilde{\Psi}_{s, m}\right\} \in \sec \emptyset\right.$ and (for all $\left.\bar{k}^{\prime \prime}\right)$ (for all $\bar{k}$ ) (exists $\left.\tilde{\Psi}_{s, r}\right)\left(\tilde{\Psi}_{s, r} \in \tilde{\Psi}_{s}\right.$ and $\left.\bigwedge_{l} \beta_{\bar{k}, l} \wedge \chi_{\bar{k}^{\prime \prime}} \models \tilde{\Psi}_{s, r}\right)$ ).

We have exploited Prop. 2 for treating the implication problems.

5. $\left(\operatorname{exists} \tilde{\Psi}_{s}\right)\left(\tilde{\Psi}_{s}=\left\{\tilde{\Psi}_{s, 1}, \ldots, \tilde{\Psi}_{s, m}\right\} \in \sec \emptyset\right.$ and

(for all $\left.\bar{k}^{\prime \prime}\right)\left(\right.$ for all "non-contradictory" $\bar{D} \in \mathscr{D}_{i-1}$ )

$\left(\right.$ exists $\left.\tilde{\Psi}_{s, r}\right)\left(\tilde{\Psi}_{s, r} \in \tilde{\Psi}_{s}\right.$ and $\left.\bigwedge_{l} \beta_{k(\bar{D}), l} \wedge \chi_{\bar{k}^{\prime \prime}} \models \tilde{\Psi}_{s, r}\right)$ ).

We have employed the correspondance between disjuncts of hist ${ }_{i-1}$ and branches, where $k(\bar{D})$ corresponds to $\bar{D}$.

6. (exists $\left.\tilde{\Psi}_{s}\right)\left(\tilde{\Psi}_{s} \in \sec _{\emptyset}\right.$ and (for all $\left.\bar{k}^{\prime \prime}\right)\left(\right.$ for all "non-contradictory" $\bar{D} \in \mathscr{D}_{i-1}$ ) $\left(\operatorname{exists} \tilde{\Psi}_{s, r}^{\bar{D}}\right)\left(\tilde{\Psi}_{s, r} \in \tilde{\Psi}_{s}^{\bar{D}}\right.$ and $\left.\left.\chi_{\bar{k}^{\prime \prime}}=\tilde{\Psi}_{s, r}^{\bar{D}}\right)\right)$.

Here $\tilde{\Psi}_{s}^{\bar{D}}$ is the version of $\tilde{\Psi}_{s}$ in the branch $\sec [\bar{D}]$. The simplifications of the adaption subprotocol preserve the applicability of the efficient implication check, as stated in Prop. 2. Bascially, this kind of assertion is checked by the violation subprotocol of the policy-adaption approach. 


\subsection{Efficiency of Policy Adaption}

Without restrictions the worst-case complexity of policy adaption is inevitably determined by the complexity of the decision problems for propositional logic and thus expected to be exponential. Exponential efforts might also be hidden in transforming sentences into disjunctive forms or even determining all prime implicants. However, queries or negated queries that consist of strict disjunctions or generate strict disjunctions, respectively, are the sole cause of branching and thus of an exponential explosion of the size of an adapted policy. Besides these general remarks, analytical complexity results on "average"-case complexity appear to be hardly obtainable and are beyond the scope of this paper. It is left open to future work to implement a prototype and to set up practical experiments. If we then aim at empirically comparing policy adaption and view generation for special cases, we will be challenged to identify the best available optimization techniques for each of the two approaches.

If we restrict on queries that are single literals and then inspect such a literal, we have to determine whether and how the atom involved occurs in one of the implicants in the current policy data. To generalize the data structure exemplified in Fig. 1 we could maintain an efficiently searchable structure of all relevant atoms, together with the set structure comprising all current implicants (then including single literals), linking an atom with all pertinent implicants.

\section{Related Work, Extensions and Conclusions}

Though the policy-adaption based approach is innovative for inference control by means of Controlled Query Evaluation, some of the underlying ideas are already implicitly present in various previous work. First of all, we observe that a mechanism for enforcing inference control can be seen as an automaton that is basically specified by its set of internal states, its state transition function and its output or reaction function. In principle, for Controlled Query Evaluation a state has to reflect both a user's history and the confidentiality policy suitably. Accordingly, in a straightforward approach, a state can just be formed by a combination of two components: a current log of the user's history and a current version of the policy. In fact, the view-based approach explicitly maintains these two components. In contrast, the policy-adaption based approach aims at representing both of the needed features within one component.

All work on state-dependent control is somehow related to our contribution, as can be seen from the following examples. The works on "enforceable security properties" [109] treat states as abstract objects, without indicating implementations. Advanced discretionary access control based on logic programming, like the Flexible Authorization Framework [8] maintains a special "done-predicate", which can be seen as a kind of a user log or as a kind of a dynamic component of the access control policy, depending on the point of view. The Dynamic Authorization Framework [3] additionally selects a current model as a dynamic policy component to determine the current semantics. Dynamic mandatory access control [2] offers to adapt security labels assigned to objects as a classification like 
"high-water marks", where classifications can be seen as a part of the access control policy. Many further examples stem from the dynamic control of workflows. Control of probabilistic inferences [7] uses a Bayesian network, which is updated after returning some piece of information to a user; the current network reflects the confidentiality requirements still to be enforced.

We demonstrated in detail that the proposed policy-adaption approach can be employed effectively for a specific situation of Controlled Query Evaluation, and we also indicated how to implement this approach such that inference control can be performed efficiently for special cases. It would be worthwhile to also consider more expressive situations, including incomplete instances and open queries. Such extensions will challenge us to transfer the current considerations to the more complex modal first-order logic. Seen from a even more general perspective, the ultimate goal of further efforts should be the following: We should aim at finding suitable combinations of the view-based approach and the policyadaption based appraoch, in order to achieve the best possible efficiency for specific situations; and maybe we could further aim at constructing an optimizer that automatically recognizes the best combination for a current situation.

\section{References}

1. Abiteboul, S., Hull, R., Vianu, V.: Foundations of Databases. Addison-Wesley, Reading (1995)

2. Bell, D.E., LaPadula, L.J.: Secure computer systems: A mathematical model, volume II. Journal of Computer Security 4(2/3), 229-263 (1996); reprint of MITRE Corporation (1974)

3. Bertino, E., Buccafurri, F., Ferrari, E., Rullo, P.: A logic-based approach for enforcing access control. Journal of Computer Security 8(2/3) (2000)

4. Biskup, J.: Security in Computing Systems - Challenges, Approaches and Solutions. Springer, Heidelberg (2009)

5. Biskup, J.: Usability confinement of server reactions: Maintaining inference-proof client views by controlled interaction execution. In: Kikuchi, S., Sachdeva, S., Bhalla, S. (eds.) DNIS 2010. LNCS, vol. 5999, pp. 80-106. Springer, Heidelberg (2010)

6. Biskup, J., Bonatti, P.A.: Controlled query evaluation for enforcing confidentiality in complete information systems. Int. J. Inf. Sec. 3(1), 14-27 (2004)

7. Chen, Y., Chu, W.W.: Protection of database security via collaborative inference detection. IEEE Trans. Knowl. Data Eng. 20(8), 1013-1027 (2008)

8. Jajodia, S., Samarati, P., Sapino, M.L., Subrahmanian, V.S.: Flexible support for multiple access control policies. ACM Trans. Database Syst. 26(2), 214-260 (2001)

9. Ligatti, J., Reddy, S.: A theory of runtime enforcement, with results. In: Gritzalis, D., Preneel, B., Theoharidou, M. (eds.) ESORICS 2010. LNCS, vol. 6345, pp. 87100. Springer, Heidelberg (2010)

10. Schneider, F.B.: Enforceable security policies. ACM Trans. Inf. Syst. Secur. 3(1), 30-50 (2000)

11. Sicherman, G.L., de Jonge, W., van de Riet, R.P.: Answering queries without revealing secrets. ACM Trans. Database Syst. 8(1), 41-59 (1983) 